\title{
CircABCB10 silencing inhibits the cell ferroptosis and apoptosis by regulating the miR-326/CCL5 axis in rectal cancer
}

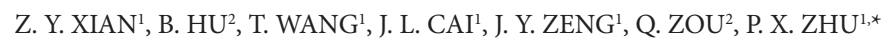 \\ ${ }^{1}$ Graceland Medical Center, The Sixth Affiliated Hospital of Sun Yat-sen University, Guangzhou, China; ${ }^{2}$ Department of Colorectal Surgery, The \\ Sixth Affiliated Hospital of Sun Yat-sen University (Gastrointestinal and Anal Hospital), Guangzhou, China
}

${ }^{*}$ Correspondence: peixuanzhu@126.com

Received October 24, 2019 / Accepted January 7, 2020

\begin{abstract}
Circular ATP binding cassette subfamily B member 10 (circABCB10) has been identified to have oncological functions in several tumors. However, the roles of circABCB10 in rectal cancer remain unknown. The expression of circABCB10, microRNA (miR)-326 and C-C motif chemokine ligand 5 (CCL5), and apoptosis related-protein was detected using quantitative real-time polymerase chain reaction or western blot, respectively. Cell survival or apoptosis was measured using cell counting kit-8 assay or flow cytometry. The accumulations of intracellular lipid reactive oxygen species (ROS) and $\mathrm{Fe} 2+$ were analyzed using C11-BODIPY dye or iron kit assay, respectively. In vivo experiments were conducted using the murine xenograft model. The interaction between miR-326 and circABCB10 or CCL 5 was confirmed by dual-luciferase reporter assay and RNA immunoprecipitation assay. CircABCB10 and CCL5 were upregulated but miR-326 was downregulated in rectal cancer. The knockdown of circABCB10 promoted rectal cancer cell ferroptosis and apoptosis in vitro as well as inhibited tumor growth in vivo. miR-326 was a target of circABCB10, and the miR-326 inhibition could partially attenuate circABCB10 deletion-induced cell ferroptosis and apoptosis. miR-326 directly interacted with CCL5, and the miR-326 inhibition suppressed cell ferroptosis and apoptosis by targeting CCL5. Besides, we observed that miR-326 was negatively regulated by circABCB10, while CCL5 was positively regulated by it, and circABCB10 served as a sponge of miR-326 to regulate the CCL5 expression in rectal cancer cells. CircABCB10 silence promoted rectal cancer cell ferroptosis and apoptosis by regulating the miR-326/CCL5 axis, suggesting a potential therapeutic target for rectal cancer therapy.
\end{abstract}

Key words: circABCB10, miR-326, CCL5, apoptosis, ferroptosis, rectal cancer

Colorectal cancer (CRC) is the third most common solid malignancy and the fourth highest leading cause of cancerrelated mortality worldwide [1]. Rectal cancer is one of the main sub-types of CRC, accounting for around 30\% of CRC, with a high risk of metastasis and relapse $[2,3]$. With the advances in the multimodality treatment of rectal cancer, locally advanced rectal cancer patients have better survival rates than patients with colon cancer, but at the expense of munificent morbidities and lower quality of life [4]. Thus, further investigations on the understanding of the molecular mechanisms involved in the pathogenesis of rectal cancer are necessary to develop ideal diagnostic or therapeutic biomarkers for early detection and treatment of rectal cancer.

Increasing evidence reports that diverse types of RNAs have emerged as important regulators in the development and progression of tumors by acting in multiple ways $[5,6]$. Circular RNAs (circRNAs) are one type of highly conserved RNA transcripts with covalently closed loop structures and are formed by the back-splicing of precursor mRNA (pre-mRNA), which make them resistant to regular mechanisms of linear RNAs decay [7, 8]. Recent studies have shown that circRNAs are dysfunctional in diverse human cancers and dysregulated circRNAs play important roles in the tumorigenesis and tumor progression in a variety of cancers, including CRC [9-11]. Circ ATP binding cassette subfamily B member 10 (circABCB10) is a novel-identified circRNA, which has been indicated to have oncological functions to promote cell proliferation and progression in breast cancer, clear cell renal cell carcinoma, and epithelial ovarian cancer [12-14]. However, the roles of circABCB10 in rectal cancer remain largely unknown.

MicroRNAs (miRNAs) are the best-characterized type of RNAs, which can interact with the complementary sequences in the 3'-untranslated region (UTR) of their target mRNAs to post-transcriptionally control gene expression [15]. It has been demonstrated that circRNAs function as 
sponges of miRNA, affect the expression of miRNAs and the competition between mRNAs $[8,16]$, thereby involving in the initiation and progression of a variety of cancers [11, 17, 18]. miR-326 is a well-recognized tumor suppressor, which has been found to be downregulated and associated with the tumorigenesis of CRC and is a potential therapeutic target for CRC [19]. C-C motif chemokine ligand 5 (CCL5) is known as a chemotactic cytokine, whose expression and secretion are regulated in T cells [20]. Although CCL5 has been initially identified as an inducer to recruit leukocytes to inflammatory sites [21], increasing findings documented the high expression of CCL5 in a variety of cancers, and abnormal CCL5 expression contributed to promote tumor development and metastasis in many cancers, including CRC $[22,23]$. However, the exact molecular mechanism of CCL5 in rectal cancer is still unclear.

Cell death is an important biological process, which can maintain homeostasis during development and disease prevention [24]. Ferroptosis and apoptosis are two modes of cell death and have been identified to play important roles in the tumorigenesis of cancers [25, 26]. Therefore, this study focused on the expression and functions of circABCB10, miR-326, and CCL5 in the ferroptosis and apoptosis of rectal cancer, and explored the potential regulatory relationship among them in cell ferroptosis and apoptosis in rectal cancer.

\section{Materials and methods}

Clinical samples. This study was approved by the Ethics Committee of The Sixth Affiliated Hospital of Sun Yat-sen University. All patients had signed informed consent before the study. Tumor tissues and adjacent non-tumor tissues from 30 rectal cancer patients who underwent surgical resection were obtained from The Sixth Affiliated Hospital of Sun Yat-sen University and were immediately stored at $-80^{\circ} \mathrm{C}$ for further analysis. All patients were diagnosed by histopathological examination and did not receive any preoperative treatment.

Cell culture. Human rectal mucosa epithelium cell line (FHC) and rectal cancer cell lines SW837 and HR-8348 were obtained from the Shanghai Academy of Life Science (Shanghai, China) and maintained in the Roswell Park Memorial Institute-1640 (RPMI-1640, Gibco, Carlsbad, CA, USA) medium supplemented with $10 \%$ fetal bovine serum (FBS, Gibco), $100 \mathrm{U} / \mathrm{ml}$ penicillin and $100 \mathrm{mg} / \mathrm{ml}$ streptomycin at $37^{\circ} \mathrm{C}$ with $5 \% \mathrm{CO}_{2}$.

Cell transfection. The short hairpin RNA (shRNA) targeting circABCB10 covalent closed junction (sh-circABCB10), shRNA scramble control (sh-NC), the small interfering RNA (siRNA) sequences targeting circABCB10 covalent closed junction (si-circABCB10, 5'-TAGAAGACCATGGGGGATGTCAAGAGCATCCCCCATGGTCTTCTATTTTTT-3'), siRNA sequences targeting CCL5 (si-CCL5, 5'-UCUUUCUGGUGAUAAAGACGAGUCUUUAUCAC-
CAGAAAGAAC-3'), siRNA negative control (si-NC) were designed by Genepharma (Shanghai, China). The miR-326 mimic (miR-326), miR-326 inhibitor (anti-miR-326), and their corresponding negative control (miR-NC and antimiR-NC) were purchased from RIBOBIO (Guangzhou, China). The transfection of plasmids or miRNAs was carried out using Lipofectamine ${ }^{\mathrm{TM}} 2000$ transfection reagent (Invitrogen, Carlsbad, CA, USA).

Quantitative real-time polymerase chain reaction (qRT-PCR). Trizol reagent (Invitrogen) was used to extract total RNA according to the standard procedure. Subsequently, isolated RNA was incubated with Rnase R (Epicentre, Madison, WI, USA), followed by the interaction with RNeasy Min Elute Cleanup Kit (Qiagen, Valencia, CA, USA). Complementary DNA (cDNA) was synthesized using the Prime Script RT Master Mix (Applied Biosystems, Foster City, CA, USA), and then quantitative PCR was carried out with SYBR Premix Ex Taq (Qiagen). Relative transcription alterations were analyzed by $2^{-\Delta \Delta \mathrm{Ct}}$ method and normalized by glyceraldehyde 3-phosphate dehydrogenase (GADPH) or U6 small nuclear B noncoding RNA (U6). The specific primer sequences were presented as follow: circABCB10: F 5'-CTAAGGAGTCACAGGAAGACATC-3', R 5'-GTAGAATCTCTCAGACTCAAGGTTG-3'; CCL5: F 5'-CCATGAAGGTCTCCGCGGCAC-3', R 5'-CCTAGCTCATCTCCAAAGAG-3'; miR-326: F 5'-CCTCTGGGCCCTTCCTCCAG-3', R 5'-GCTGTCAACGATACGCTACCTA-3'; GADPH: F 5'-GATATTGTTGCCATCAATGAC-3', R 5'-TTGATTTTGGAGGGATCTCG-3'; U6: F 5'-CTCGCTTCGGCAGCACA-3', R 5'-ACGCTTCACGAATTTGCGT-3'.

Cell viability assay. Transfected SW837 and HR-8348 cells were seeded into the wells of a 96-well plate. After stimulation with erastin (Selleck Chemicals, Houston, TX, USA; 0-40 $\mu \mathrm{M}$ ) for $24 \mathrm{~h}, 10 \mu \mathrm{l}$ of cell counting kit-8 (CCK-8) solution (Beyotime, Shanghai, China) was added into each well and incubated for another $2 \mathrm{~h}$. Finally, the reading of the optical density (OD) was detected on a microplate reader at $450 \mathrm{~nm}$ to assess the survival rate.

Lipid reactive oxygen species (ROS) measurement. Transfected cells were treated with $5 \mu \mathrm{M}$ erastin for $24 \mathrm{~h}$, and then were resuspended in medium with $10 \%$ FBS (Gibco). Immediately, $10 \mu \mathrm{M}$ C11-BODIPY (Thermo Fisher Scientific, Inc., Waltham, MA, USA) was added to the cell suspension and incubated for $30 \mathrm{~min}$ at $37^{\circ} \mathrm{C}$ in the dark. After washing with PBS twice, the fluorescence intensity of the samples was measured by using a flow cytometer.

Iron assay. The level of intracellular $\mathrm{Fe}^{2+}$ was detected by the iron assay kit (Abcam, Cambridge, MA, USA) following the standard procedure. In brief, transfected cells were treated with $5 \mu \mathrm{M}$ erastin for $24 \mathrm{~h}$. Cells were collected and washed with cold PBS and then homogenized in 4-10 volumes of the iron assay buffer. After removing the insoluble material, $5 \mu \mathrm{l}$ iron reducer was added to each well before mixing, followed by incubation for $30 \mathrm{~min}$ at $37^{\circ} \mathrm{C}$ in the dark. Subsequently, each well was added with $100 \mu$ of the iron probe and mixed, 
and then incubated for $60 \mathrm{~min}$ at $37^{\circ} \mathrm{C}$ in dark conditions. The OD was detected at $593 \mathrm{~nm}$ using a colorimetric microplate reader. Besides, total protein concentration in $50 \mu \mathrm{l}$ samples was quantified using the bicinchoninic acid assay, and the level of $\mathrm{Fe}^{2+}$ was assessed with the following equation: level $=$ volume $(\mathrm{nmol} / \mu \mathrm{l}) /$ concentration $(\mathrm{mg} / \mu \mathrm{l})$.

Cell apoptosis assay. Annexin V-fluorescein isothiocyanate (FITC)/propidium iodide (PI) apoptosis detection kit (BD Biosciences, San Jose, CA, USA) was used to detect apoptotic cells following the standard protocol. Transfected cells were resuspended in binding buffer, followed by staining with $5 \mu \mathrm{l}$ FITC annexin V and $10 \mu \mathrm{l}$ PI. Finally, the apoptotic cells were measured by FlowJo software.

Western blot analysis. Transfected cells were collected and then lysed using the radio-immunoprecipitation assay (RIPA) buffer (Beyotime). Cell lysates were separated on 10\% sodium dodecyl sulfate polyacrylamide gel electrophoresis, transferred onto a polyvinylidene fluoride membrane, and blocked with 5\% non-milk. Subsequently, the membranes were incubated with primary antibodies against B-cell lymphoma-2 (Bcl-2, 1:500, ab692, Abcam), Bcl2-associated $\mathrm{X}$ protein (Bax, 1:1000, ab32503, Abcam), CCL5 (1:1000, ab9679, Abcam) and $\beta$-Actin (1:1000, 4967, Cell Signaling Technology, Boston, MA, USA), followed by incubation with secondary HRP-conjugated antibody (1:1000, ab9482, Abcam). Finally, signals were visualized using the chemiluminescence chromogenic substrate (Beyotime).

In vivo experiments. $\mathrm{BALB} / \mathrm{c}$ nude mice (female, $4-6$ weeks of age, $\mathrm{N}=6$ ) were used to perform the xenograft assays. HR-8348 cells were stably transfected with lentivirus containing sh-circ-ABCB10 or sh-NC and then subcutaneously injected into the flanks of the nude mice. After one week following the inoculation, the tumor size was examined every week and the tumor volume was calculated. After 4 weeks, all mice were sacrificed and tumor masses were weighted and harvested for further molecular analysis. This study was approved by the Animal Research Committee of The Sixth Affiliated Hospital of Sun Yat-sen University and undertaken in accordance with the guidelines of the National Animal Care and Ethics Institution.

Dual-luciferase reporter assay. The wild-type (WT) or mutant (MUT) circ-ABCB10/CCL5 3'UTR possessing miR-326 binding sequences were amplified and cloned into the pmirGLO Vector (Promega, Shanghai, China). Then, SW837 and HR-8348 cells were co-transfected with the constructed vectors and miR-326 or miR-NC using Lipofectamine 2000 reagent (Invitrogen) and incubated for 48 h. Finally, a dual-luciferase reporter assay kit (Promega) was used to measure the relative luciferase activity.

RNA immunoprecipitation (RIP) assay. RIP assay was performed with the Magna RIP Kit (Millipore, Billerica, MA, USA). SW837 and HR-8348 cells transfected with miR-326 or miR-NC were pelleted and lysed in RIP buffer (Millipore), and then the lysate was incubated with magnetic beads coated with human anti-Ago2 or IgG antibody. Subsequently, the enrichment of circ-ABCB10 or CCL5 was measured by qRT-PCR as described above, respectively.

Statistical analysis. All statistical data from triplicate repeat independent experiment were expressed as a mean \pm standard deviation (SD) and analyzed using GraphPad Prism 7 software (GraphPad Inc., San Diego, CA, USA). The differences in different groups were analyzed by Student's $\mathrm{t}$-test or one-way analysis of variance (ANOVA). The correlation analysis among circ-ABCB10, miR-326, and CCL5 was performed using Spearman's rank correlation. A p-value $<0.05$ presented statistically significant.

\section{Results}

CircABCB10 is upregulated in rectal cancer and its depletion regulates erastin-induced ferroptosis in rectal cancer. To explore the roles of circABCB10 in rectal cancer, we firstly detected the expression of circABCB10 in 30 paired rectal cancer patients, and results showed circABCB10 was higher in rectal cancer tissues compared to the normal tissues (Figure 1A). Similarly, the qRT-PCR analysis also indicated an upregulation of circABCB10 in rectal cancer cell lines SW837 and HR-8348 compared with the rectal mucosa epithelium cell line (FHC) (Figure 1B). Erastin is a classical small-molecule inducer of ferroptosis [27]. To detect whether circABCB 10 could regulate ferroptosis, circABCB10 was inhibited in SW837 and HR-8348 cells using siRNA sequences. As expected, circABCB10 was significantly reduced in SW837 and HR-8348 cells (Figure 1C). After treatment with erastin $(0-40 \mu \mathrm{M})$ for $24 \mathrm{~h}$, CCK-8 assay indicated erastin-induced cell death in SW837 and HR-8348 cells in a dose-dependent manner and circABCB10 depletion significantly increased erastin-induced ferroptosis both in SW837 and HR-8348 cells (Figures 1D, 1E), suggesting that circABCB10 might be an important regulator of ferroptosis in rectal cancer cells.

CircABCB10 depletion promotes rectal cancer cell ferroptosis and apoptosis in vitro as well as inhibits tumor growth in vivo. Ferroptosis is involved in the generation of iron-dependent ROS, thus we investigated the accumulation of lipid ROS and $\mathrm{Fe}^{+}$, which are two essential factors in triggering ferroptosis [27], in rectal cancer cells treated with $5 \mu \mathrm{M}$ erastin for $24 \mathrm{~h}$. We found that circABCB10 depletion significantly promoted the accumulation of intracellular lipid ROS in SW837 and HR-8348 cells (Figure 2A). Furthermore, the knockdown of circABCB10 also increased the intracellular concentrations of $\mathrm{Fe}^{2+}$ in SW837 and HR-8348 cells (Figure 2B), indicating circABCB10 depletion induced sensitivity to ferroptosis in rectal cancer cells. Besides that, we observed that circABCB10 depletion induced cell apoptosis in SW837 and HR-8348 cells (Figure 2C). Moreover, western blot showed circABCB10 silence increased the level of Bax, but decreased the level of Bcl-2 in SW837 and HR-8348 cells (Figure 2D), further implying circABCB10 silence induced cell apoptosis in rectal cancer. 


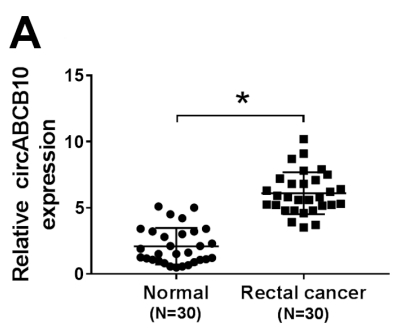

D

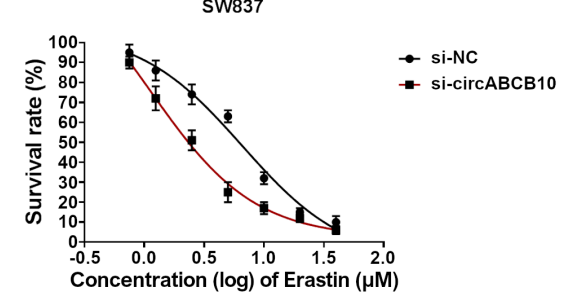

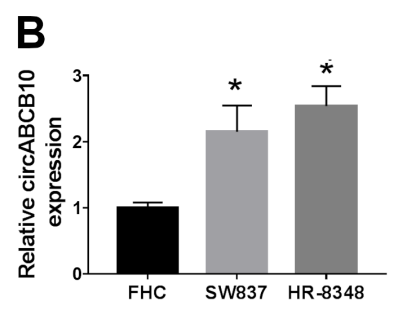
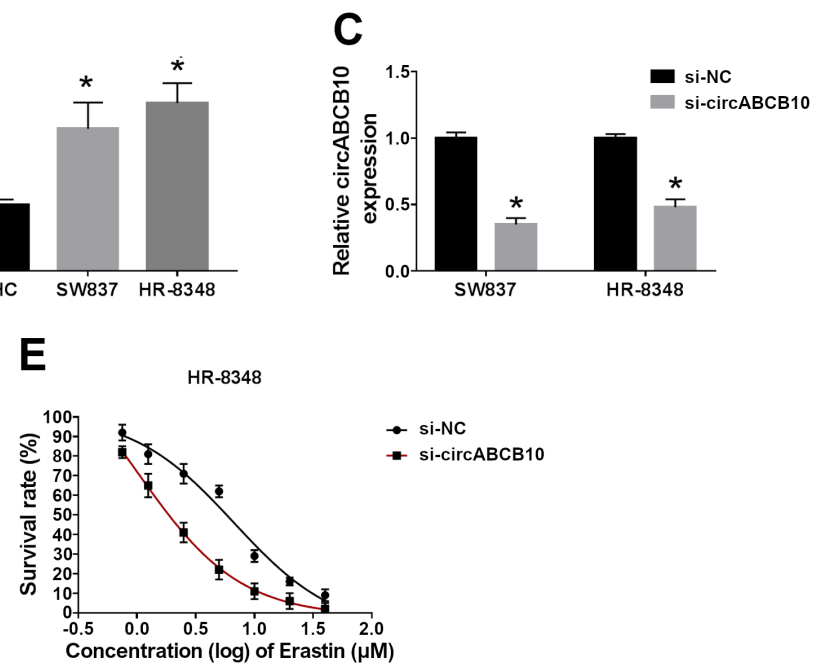

Figure 1. CircABCB10 is upregulated in rectal cancer and circABCВ10 depletion regulates erastin-induced ferroptosis in rectal cancer. $A$, $B$ ) The expression of circABCB10 was detected using $\mathrm{qRT}-\mathrm{PCR}$ in rectal cancer tumor tissues, normal tissues, rectal cancer cell lines SW837 and HR-8348, as well as rectal mucosa epithelium cell line (FHC; Student's $t$ test). circABCB10 was inhibited in SW837 and HR-8348 cells using siRNA sequences. C) The interference efficiency was measured using qRT-PCR (Student's t test). D, E) SW837 and HR-8348 cells were treated with erastin (0-40 $\mu M)$ for $24 \mathrm{~h}$ and cell death was assayed using a CCK-8 kit (Student's $t$ test). ${ }^{*} \mathrm{p}<0.05$
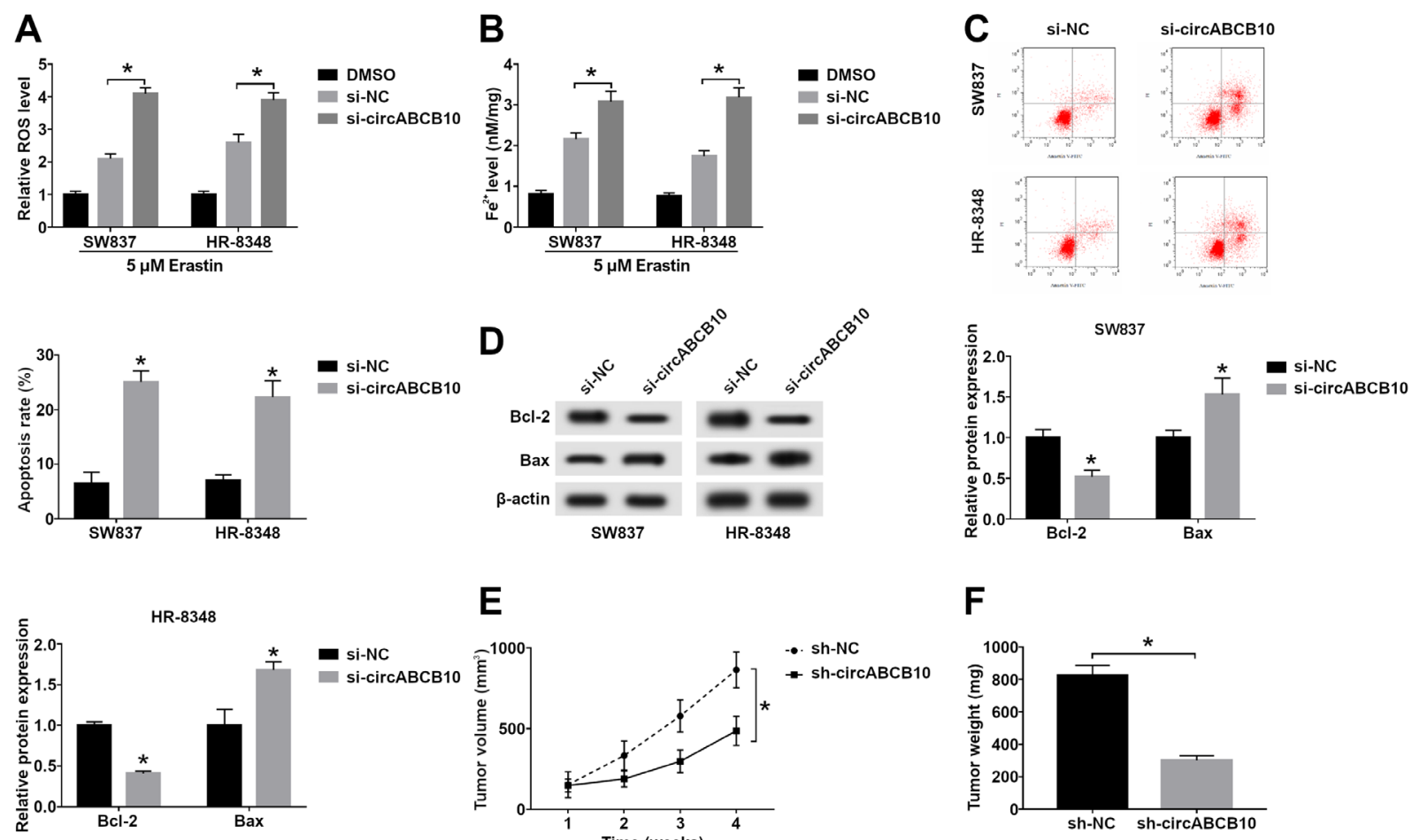

E
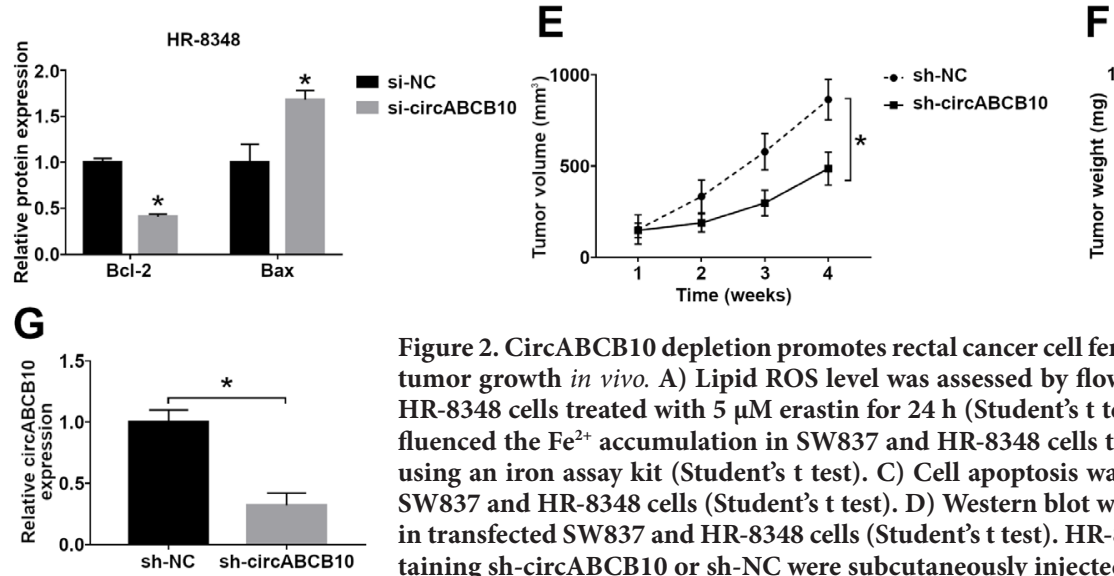

Figure 2. CircABCB10 depletion promotes rectal cancer cell ferroptosis and apoptosis in vitro as well as inhibits tumor growth in vivo. A) Lipid ROS level was assessed by flow cytometry using C11-BODIPY in SW837 and HR-8348 cells treated with $5 \mu \mathrm{M}$ erastin for $24 \mathrm{~h}$ (Student's $\mathrm{t}$ test). B) The expression levels of circABCB10 influenced the $\mathrm{Fe}^{2+}$ accumulation in SW837 and HR-8348 cells treated with $5 \mu \mathrm{M}$ erastin for $24 \mathrm{~h}$ were detected using an iron assay kit (Student's t test). C) Cell apoptosis was analyzed using flow cytometry in transfected SW837 and HR-8348 cells (Student's $t$ test). D) Western blot was used to determine the levels of Bax and Bcl-2 in transfected SW837 and HR-8348 cells (Student's $t$ test). HR-8348 cells stably transfected with lentivirus containing sh-circABCB10 or sh-NC were subcutaneously injected into the flanks of the nude mice to perform in vivo experiments. E) Tumor volumes were calculated every week (Student's t test). F) Mice were euthanized on day 28 after infection, and then tumor masses were excised and weighed (Student's $t$ test). G) The expression of circABCB10 in excised tumor masses was determined using $\mathbf{q R T}-\mathrm{PCR}$ (Student's $\mathrm{t}$ test). ${ }^{*} \mathbf{p}<0.05$ 
After that, HR-8348 cells stably transfected with lentivirus containing sh-circABCB10 or sh-NC were subcutaneously injected into the flanks of the nude mice to perform in vivo experiments. We observed that circABCB10 depletion markedly impaired tumor volume, weight, and inhibited circABCB10 expression in the sh-circABCB10 group (Figures $2 \mathrm{E}-2 \mathrm{G}$ ). Taken together, our results demonstrated that circABCB10 was associated with cell apoptosis, ferroptosis, and tumor growth in vivo and in vitro, suggesting an oncogenic role of circABCB10 in rectal cancer progression.

CircABCB10 is a sponge of miR-326 and regulates miR-326 expression. To detect the potential pathways by which circABCB10 regulated cell ferroptosis and apoptosis in rectal cancer, the potential miRNA targets of circABCB10 were searched using the bioinformatics tool starBase program and $\mathrm{miR}-326$ was predicted to contain putative binding sites of circABCB10 (Figure 3A). To verify the bioinformatics-based prediction, a dual-luciferase activity assay was conducted and results showed miR-326 overexpression dramatically reduced the luciferase activity of WT circABCB10 reporter vector, but not MUT circABCB10 reporter vector in SW837 and HR-8348 cells (Figure 3B). Furthermore, RIP assay using anti-Ago2 antibody also confirmed that miR-326 interacted with circABCB10 in SW837 and HR-8348 cells due to the enrichment of circABCB10 (Figure 3C). In addition,
qRT-PCR exhibited that the knockdown of circABCB10 promoted miR-326 expression in SW837 and HR-8348 cells (Figure 3D). These data suggested circABCB10 as a sponge of miR-326 in rectal cancer cells. In the meanwhile, miR-326 was found to be decreased in rectal cancer tissues and cell lines (Figures $3 \mathrm{E}, 3 \mathrm{~F}$ ), and a negative correlation between miR-326 and circABCB10 was observed in tumor tissues (Figure $3 \mathrm{G}$ ), suggesting miR-326 might be involved in circABCB10-induced regulation on rectal cancer cells.

CircABCB10 depletion promotes rectal cancer cell ferroptosis and apoptosis by interacting with miR-326. Based on the relationship between circABCB10 and miR-326, we explored whether the circABCB10/miR-326 axis was responsible for rectal cancer cell ferroptosis and apoptosis. First, SW837 and HR-8348 cells were transfected with the anti-miR-326 and anti-miR-NC, and a significantly decreased miR-326 was found in SW837 and HR-8348 cells (Figure 4A). Next, SW837 and HR-8348 cells were transfected with anti-miR-NC, anti-miR-326, si-circABCB10+anti-miR$\mathrm{NC}$, or si-circABCB10+anti-miR-326 to conduct the rescue assay. Subsequently, results indicated miR-326 inhibition significantly decreased erastin-induced ferroptosis (Figure $4 \mathrm{~B}$ ), and the accumulation of intracellular lipid ROS (Figure 4C) as well as $\mathrm{Fe}^{2+}$ (Figure 4D) both in SW837 and HR-8348 cells. Furthermore, miR-326 inhibition also suppressed

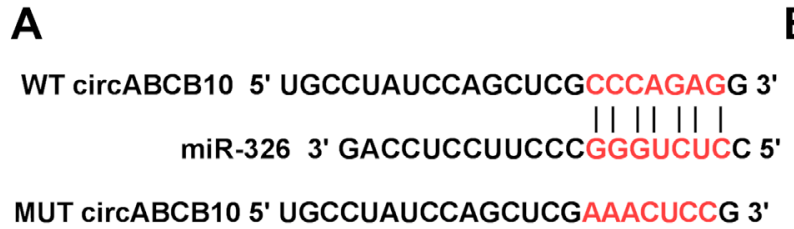

C

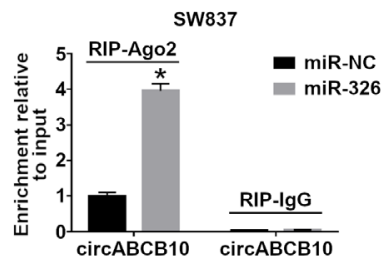

E

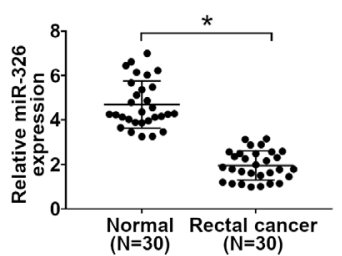

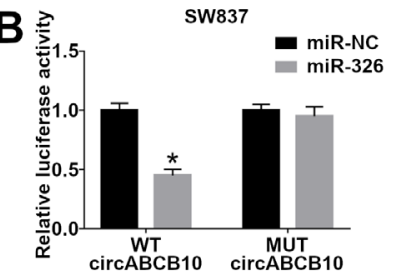

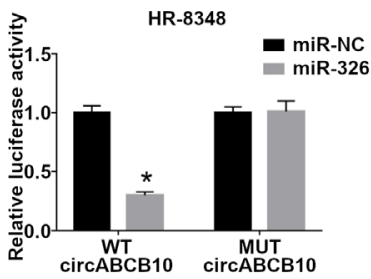

D

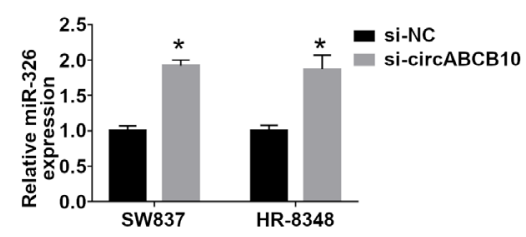

$\mathbf{F}$

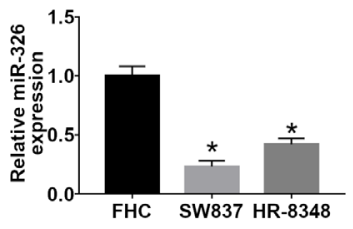

$\mathbf{G}$

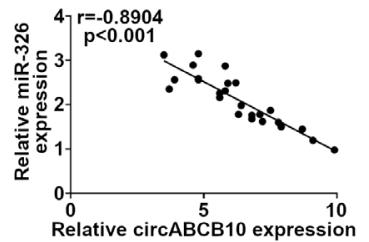

Figure 3. circABCB10 is a sponge of miR-326 and regulates miR-326 expression. $\mathrm{A}$ ) The putative binding sites between circABCB10 and miR-326 were listed. B) Luciferase activity was measured in SW837 and HR-8348 cells co-transfected with WT circABCB10 or MUT circABCB10 and miR-NC or miR-326 (Student's t test). C) The enrichment of circABCB10 was detected in SW837 and HR-8348 cells transfected with miR-NC or miR-326 after RIP (Student's t test). D) The level of miR-326 was assessed using qRT-PCR in SW837 and HR-8348 cells transfected with si-NC or si-circABCB10 (Student's $\mathrm{t}$ test). E, F) qRT-PCR was applied to measure the level of miR-326 in rectal cancer tumor tissues and cell lines (Student's test). G) The correlation between circABCB10 and miR-326 was analyzed using Spearman's rank correlation. ${ }^{*} \mathrm{p}<0.05$ 

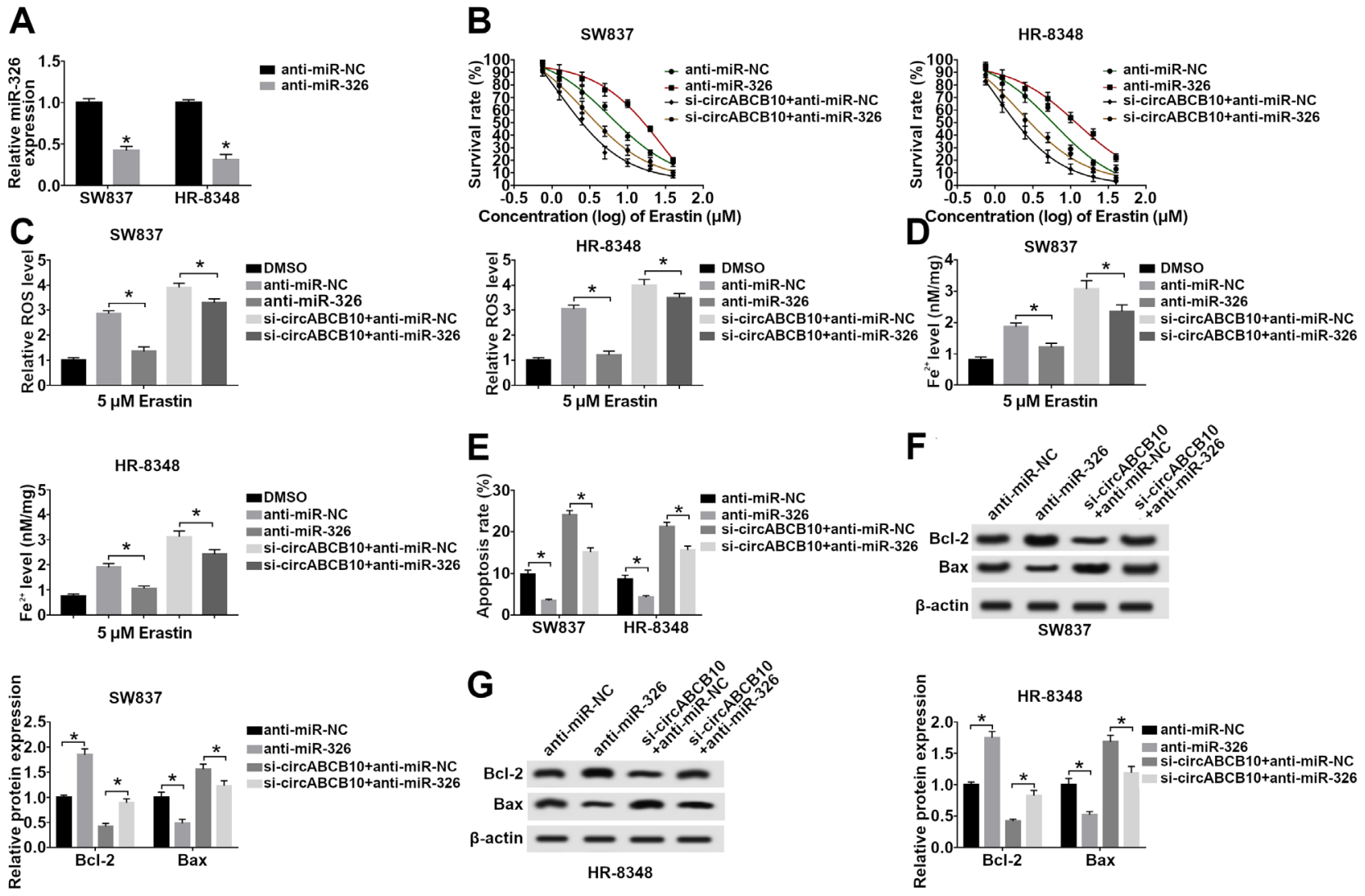

Figure 4. circABCB10 depletion promotes rectal cancer cell ferroptosis and apoptosis by interacting with miR-326. SW837 and HR-8348 cells were transfected with anti-miR-NC or anti-miR-326. A) The interference efficiency was measured using qRT-PCR (Student's t test). B) Cell death was assayed using a CCK-8 kit in SW837 and HR-8348 cells treated with erastin (0-40 $\mu \mathrm{M})$ for 24 h (One-way ANOVA). C) Lipid ROS level was assessed by flow cytometry using C11-BODIPY in SW837 and HR-8348 cells treated with $5 \mu$ M erastin for 24 h (One-way ANOVA). D) The expression levels of the $\mathrm{Fe}^{2+}$ accumulation in SW837 and HR-8348 cells treated with $5 \mu \mathrm{M}$ erastin for $24 \mathrm{~h}$ were detected using an iron assay kit (One-way ANOVA). E) Cell apoptosis was analyzed using flow cytometry in transfected SW837 and HR-8348 cells (One-way ANOVA). F, G) Western blot was used to determine the level of Bax and Bcl-2 in transfected SW837 and HR-8348 cells (One-way ANOVA). ${ }^{*}$ p $<0.05$

SW837 and HR-8348 cell apoptosis, demonstrated by the decreased apoptotic cells (Figure 4E) and Bax expression (Figures 4F, 4G), as well as the increased expression of Bcl-2 (Figures 4F, 4G). Meanwhile, the rescue assay showed miR-326 inhibition could partially reverse circABCB10 depletion-induced promotion on cell ferroptosis (Figures $4 \mathrm{~B}-4 \mathrm{D}$ ) and apoptosis (Figures 4E-4G) in SW837 and HR- 8348 cells. These results suggested circABCB10 depletion promoted rectal cancer cell ferroptosis and apoptosis by serving as a sponge of miR-326.

CCL5 is a target of miR-326 and circABCB10 indirectly regulates CCL5 by binding to miR-326. We further elucidated the mechanisms underlying the circABCB10/miR-326 mediated ferroptosis and apoptosis of rectal cancer cells, thus the target genes of miR-326 were searched. Using the bioinformatics tool TargetScan program, we found CCL5 contained the putative binding sequences of miR-326 (Figure 5A). To confirm the interaction, we performed a dual-luciferase reporter assay and found the luciferase activity of the WT-CCL5 reporter was obviously repressed, but there was no notable change in MUT-CCL5 reporter after overexpression of miR-326 in SW837 and HR-8348 cells (Figure 5B). In the meanwhile, using a RIP assay, we evaluated the CCL5 level in SW837 and HR-8348 cells after miR-326 immunoprecipitation using IgG as controls and found CCL5 was enriched after miR-326 immunoprecipitation in SW837 and HR-8348 cells, further suggesting the interaction between miR-326 and CCL5 (Figure 5C). Besides that, we demonstrated circABCB10 depletion inhibited CCL5 expression using qRT-PCR and western blot assay, while this inhibition could be rescued by the inhibition of miR-326 in SW837 and HR-8348 cells (Figures 5D, 5E). Therefore, a circABCB10/miR-326/CCL5 axis was identified in rectal cancer cells.

miR-326 inhibition inhibits cell ferroptosis and apoptosis by interacting with CCL5 in rectal cancer. Given that miR-326 targeted CCL5, we hypothesized that CCL5 might influence miR-326 induced regulation on cell 
A

\section{WT CCL5 3'UTR 5' CUCCUUGAAGGGCCCAGAGAUU 3' | |||||||| $\mid$ miR-326 3' GACCUCCUUCCCGGGUCUCC 5'} MUT CCL5 3'UTR 5' CUCCUUUCCAAAGgGgAAGAUU 3'
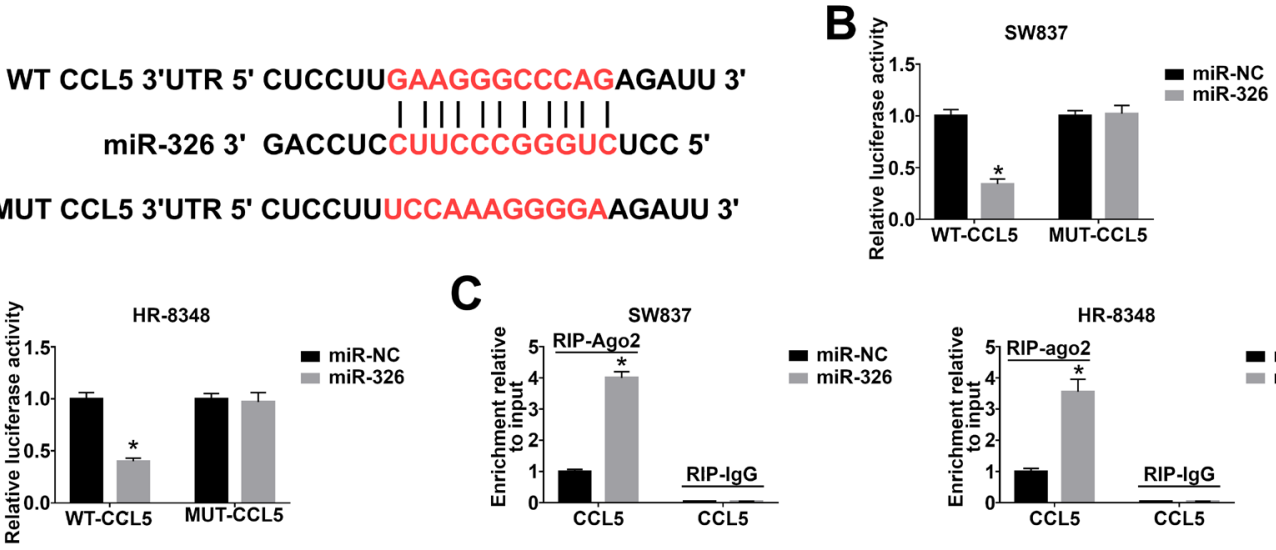

C
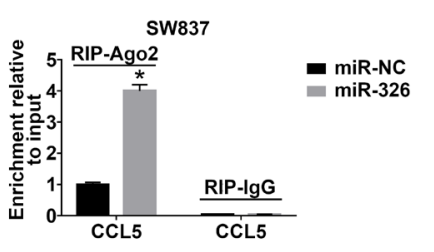

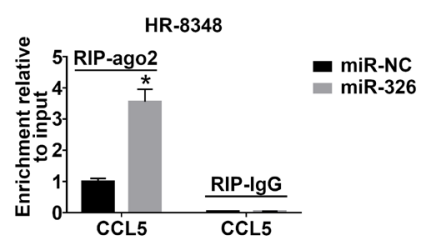

D
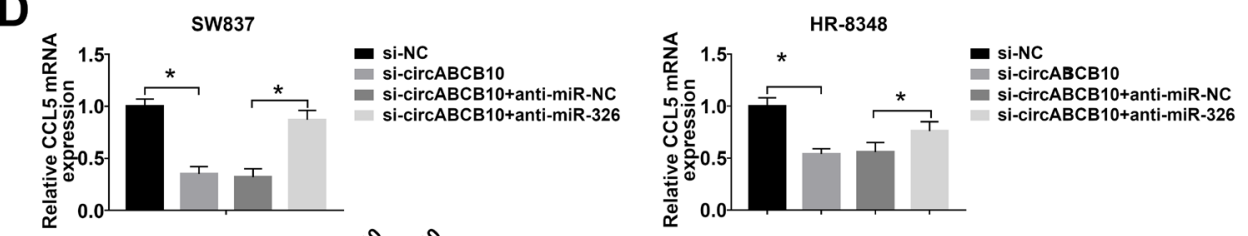
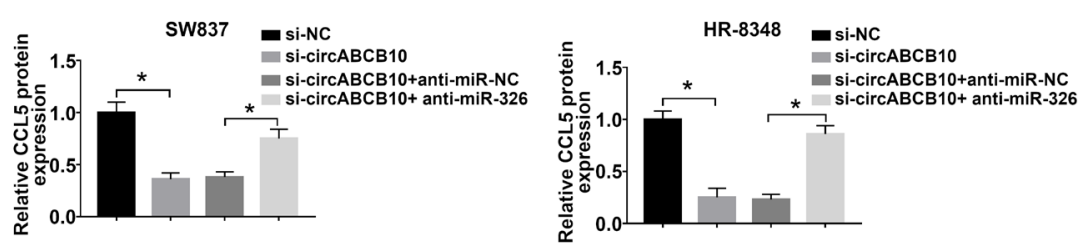

Figure 5. circABCB10 indirectly regulates CCL5 by binding to miR-326. A) The putative binding sites between CCL5 and miR-326 were presented. B) Luciferase activity was measured in SW837 and HR-8348 cells co-transfected with WT-CCL5 or MUT-CCL5 and miR-NC or miR-326 (Student's t test). C) The enrichment of CCL5 was detected in SW837 and HR-8348 cells transfected with miR-NC or miR-326 after RIP (Student's t test). SW837 and HR-8348 cells were transfected with si-NC, si-circABCB10, si-circABCB10+anti-miR-NC, or si-circABCB10+anti-miR-326. D, E) The level of CCL5 at mRNA and protein level was determined using QRT-PCR and western blot assay in SW837 and HR-8348 cells after treatment (One-way ANOVA). ${ }^{*} \mathrm{p}<0.05$

ferroptosis and apoptosis in rectal cancer. First, we determined CCL5 expression at mRNA and protein levels, and results showed CCL5 was high in rectal cancer tumor tissues and cell lines compared with the controls (Figures 6A-6D). Moreover, a negative correlation between CCL5 and miR-326 (Figure 6E), and a positive correlation between CCL5 and circABCB10 (Figure 6F) were observed, indicating the regulatory relationship among the circABCB10, miR-326, and CCL5. Subsequently, CCL5 was suppressed in SW837 and HR-8348 cells using siRNA sequences, and CCL5 expectedly decreased in SW837 and HR-8348 cells at mRNA and protein levels (Figures 6G, 6H).

After that, functional experiments were performed. We found CCL5 silence increased erastin-induced ferroptosis (Figure 7A), and the intracellular accumulation of intracellular lipid ROS (Figure 7B) and $\mathrm{Fe}^{2+}$ (Figure 7C) both in SW837 and HR-8348 cells. Additionally, CCL5 silence also promoted cell apoptosis of SW837 and HR-8348 cells (Figure 7D), and western blot analysis indicated CCL5 silence reduced the level of Bcl-2, but enhanced the level of Bax in SW837 and HR-8348 cells (Figures 7E, 7F), further demonstrating CCL5 silence induced apoptosis of SW837 and HR-8348 cells. However, the rescue assay showed CCL5 silence could partially overturn miR-326 inhibition-induced repression on cell ferroptosis (Figures $7 \mathrm{~A}-7 \mathrm{C}$ ) and apoptosis (Figures 7D-7F) in SW837 and HR-8348 cells. Altogether, miR-326 regulated cell ferroptosis and apoptosis by interacting with CCL5 in rectal cancer.

\section{Discussion}

Recently, with the development of high-throughput technologies, emerging evidence indicates the pivotal role of circRNAs in the tumorigenesis of cancers [28]. Until now, many circRNAs have been identified to be involved in the development and progression of CRC. For example, circ_0026344 suppressed cell migration, invasion, and epithelial-mesenchymal transition (EMT) process by directly 
A

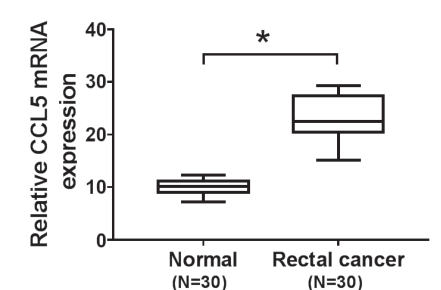

D

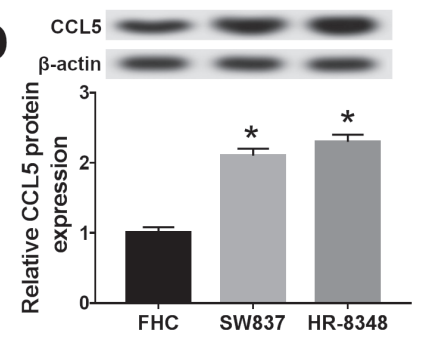

G

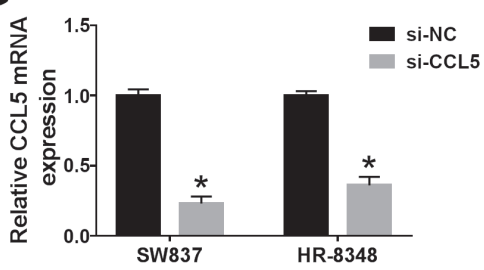

B

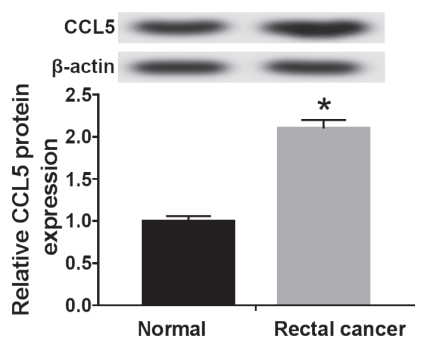

$\mathbf{E}$

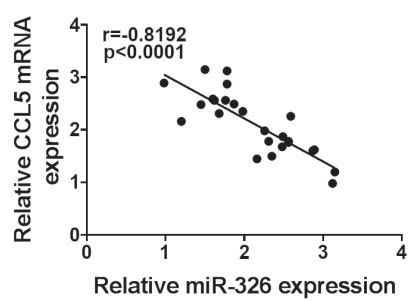

H

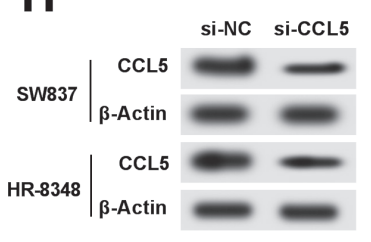

C

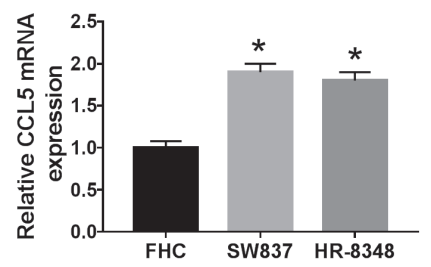

$\mathbf{F}$
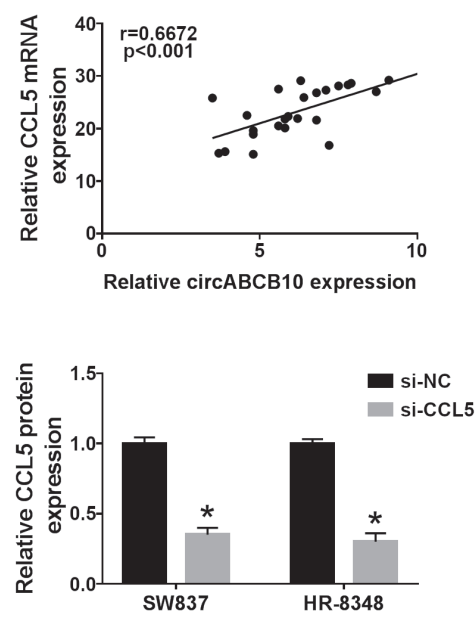

Figure 6. CCL5 is upregulated in rectal cancer. (A-D) The expression of CCL5 was detected in rectal cancer tumor tissues and cell lines at mRNA and protein levels using qRT-PCR or western blot assay (Student's t test). (E, F) The correlation between CCL5 and miR-326 or circABCB10 was assessed using Spearman's rank correlation. $(\mathrm{G}, \mathrm{H})$ The expression of CCL5 was measured in SW837 and HR-8348 cells transfected with si-CCL5 or si-NC using qRT-PCR and western blot assay (Student's $\mathrm{t}$ test). ${ }^{\star} \mathrm{p}<0.05$.

interacting with miR-183 in CRC [29]. Circ_0000218 performed carcinogenic effects to regulate cell proliferation and metastasis in CRC through the miR-139-3p/RAB1A axis [30]. Circ_0021977 served as a tumor suppressor to inhibit cell metastasis and proliferation in CRC by regulating the expression of p21 and p53 through miR-10b-5p [31]. Therefore, circRNAs may be promising candidates for the progression and therapy of CRC.

Cell death is a primary element of cellular metabolism and is necessary for the development and prevention of disease [32]. Previous evidence documented that apoptosis was the only regulated form of cell death [25], but recent studies have indicated several non-apoptotic cell death pathways, such as ferroptosis [27]. Ferroptosis is an oxidative, iron-dependent mode of non-apoptotic cell death, which induces the inactivation of glutathione and the production of toxic lipid ROS. Herein, we explored circABCB10-related epigenetic regulation in apoptosis and ferroptosis in rectal cancer. Firstly, circABCB10 was found to be elevated in rectal cancer tumor tissues and cell lines. After decreased the level of circABCB10 in rectal cancer cells using siRNA sequences, and treated with erastin, we found erastin-induced cell death in rectal cancer cells in a dose-dependent manner and circABCB10 depletion significantly increased erastin-induced ferroptosis rectal cancer cells. At the same time, the obvious accumulation of intracellular lipid ROS and $\mathrm{Fe}^{2+}$ was observed in rectal cancer cells. All the results indicated circABCB10 depletion induced sensitivity to ferroptosis in rectal cancer cells. Subsequently, circABCB10 depletion also was demonstrated to induce cell apoptosis in rectal cancer cells, reflected by the promotion of apoptotic cells and Bax expression, as well as the decreased level of $\mathrm{Bcl}-2$. Besides that, in vivo experiments suggested that circABCB10 silence inhibited rectal cancer tumor growth in vivo.

Both circRNAs and miRNAs have dynamic roles in transcriptional and translational regulation, and interactions between circRNAs and miRNAs are involved in the progression of diverse cancers, including CRC [30, 31]. Thus, we further investigated the potential target miRNAs in binding and regulating circRNAs in rectal cancer. Here, we presented strong evidence showing that circABCB10 was a sponge of miR-326 and negatively regulated miR-326 expression. miR-326 is a well-recognized tumor suppressor and associated with the tumorigenesis of CRC [19]. In this 
A

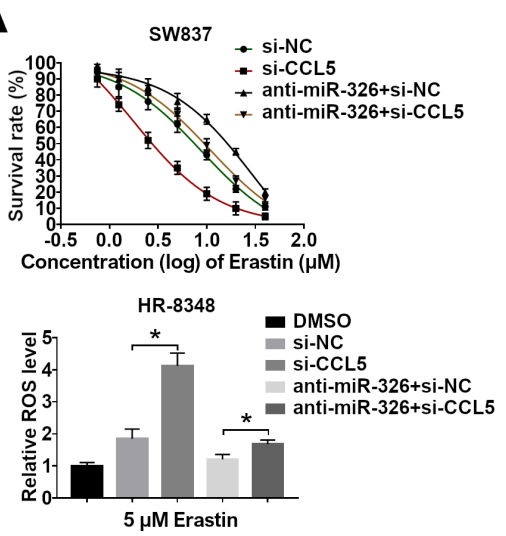

D

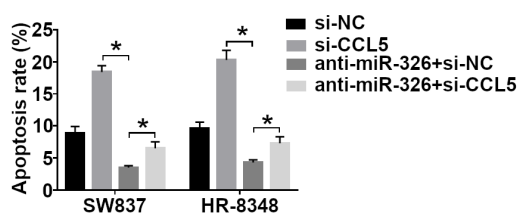

$\mathbf{F}$

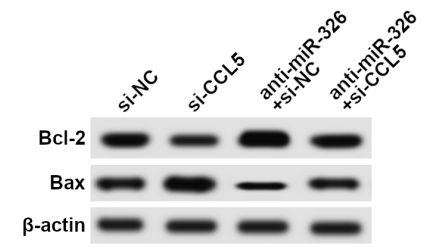

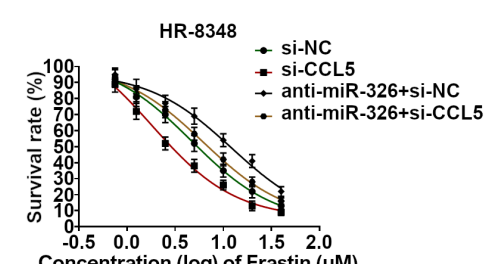

$c$
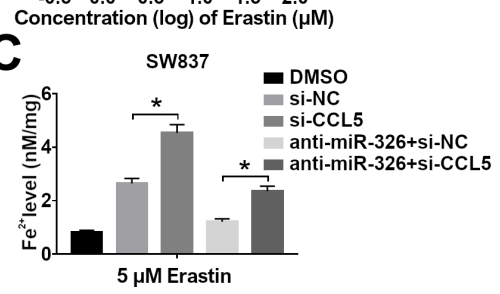

E

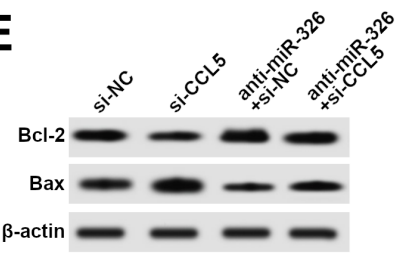

$\beta$-actin
B
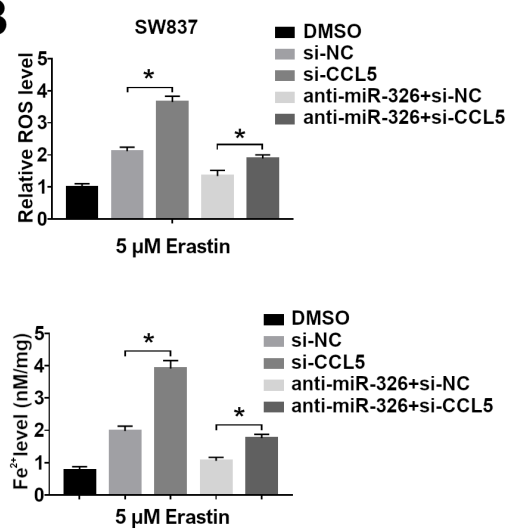

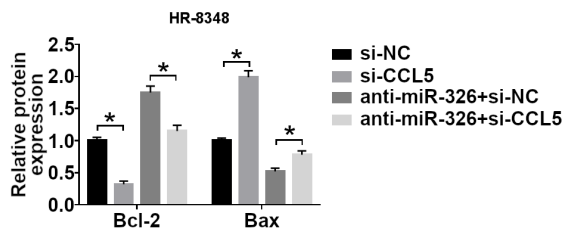

Figure 7. miR-326 inhibition inhibits cell ferroptosis and apoptosis by interacting with CCL5 in rectal cancer. A) Cell death was assayed using a CCK-8 kit in SW837 and HR-8348 cells treated with erastin (0-40 $\mu \mathrm{M})$ for $24 \mathrm{~h}$ (One-way ANOVA). B) Lipid ROS level was calculated by flow cytometry using C11-BODIPY in SW837 and HR-8348 cells treated with $5 \mu \mathrm{M}$ erastin for $24 \mathrm{~h}$ (One-way ANOVA). C) The expression levels of Fe ${ }^{2+}$ accumulation in SW837 and HR-8348 cells treated with $5 \mu \mathrm{M}$ erastin for $24 \mathrm{~h}$ were detected using an iron assay kit (One-way ANOVA). D) Cell apoptosis was analyzed using flow cytometry in transfected SW837 and HR-8348 cells (One-way ANOVA). E, F) Western blot was used to determine the levels of Bax and Bcl-2 in transfected SW837 and HR-8348 cells (One-way ANOVA). ${ }^{*}$ p $<0.05$

study, miR-326 was downregulated in rectal cancer, and functional experiments suggested miR-326 performed antitumor functions by promoting cell ferroptosis and apoptosis in rectal cancer cell lines. Moreover, rescue assay implied miR-326 inhibition could partially reverse circABCB10 depletion-induced promotion on cell ferroptosis and apoptosis in rectal cancer cells.

It is well known that miRNAs play crucial roles in diverse physiological and pathological processes, such as cell proliferation, apoptosis, metastasis, and so on, by directly regulating target gene expression in various tumors [33]. For instance, miR-520 restrained cell proliferation and invasion in CRC by directly interacting with AEG-1 [34]. miR-3174 performed carcinogenic roles in rectal cancer to accelerate cell proliferation and cell cycle by targeting PCBD2 [35]. Here, we next explored the possible target genes of miR-326 using bioinformatics tools, and CCL5 was a target of miR-326 and was negatively regulated by miR-326 in rectal cancer. CCL5 is a chemotactic cytokine and has been reported to be associated with the development and progression of rectal cancer [36]. In the current study, CCL5 was upregulated in rectal cancer and functioned carcinogenic effects to inhibited cell ferroptosis and apoptosis in rectal cancer cells. Furthermore, we also found CCL5 silence could partially overturn miR-326 inhibition-induced anti-ferroptosis and anti-apoptosis in rectal cancer cells. Additionally, we also observed that CCL5 was positively regulated by circABCB10, and circABCB10 served as an endogenous sponge of miR-326 to regulate the CCL5 expression in rectal cancer cells. Thus a circABCB10/ miR-326/CCL5 regulatory network was identified in rectal cancer cells.

In conclusion, our results demonstrated circABCB10 performed carcinogenic functions to suppress ferroptosis and apoptosis in rectal cancer by regulating miR-326/CCL5 axis, suggesting a novel insight for tumor progression and a promising therapeutic target of rectal cancer therapy. 


\section{References}

[1] FERLAY J, SOERJOMATARAM I, DIKSHIT R, ESER S, MATHERS $C$ et al. Cancer incidence and mortality worldwide: sources, methods and major patterns in GLOBOCAN 2012. Int J Cancer 2015; 136: E359-386. https://doi. org/10.1002/ijc.29210

[2] BAILEY CE, HU CY, YOU YN, BEDNARSKI BK, RODRIGUEZ-BIGAS MA et al. Increasing disparities in the agerelated incidences of colon and rectal cancers in the United States, 1975-2010. JAMA Surg 2015; 150: 17-22. https://doi. org/10.1001/jamasurg.2014.1756

[3] CONDE-MUINO R, CUADROS M, ZAMBUDIO N, SEGURA-JIMENEZ I, CANO $\mathrm{C}$ et al. Predictive Biomarkers to Chemoradiation in Locally Advanced Rectal Cancer. Biomed Res Int 2015; 2015: 921435. https://doi. org/10.1155/2015/921435

[4] GARCIA-AGUILAR J, GLYNNE-JONES R, SCHRAG D. Multimodal Rectal Cancer Treatment: In Some Cases, Less May Be More. Am Soc Clin Oncol Educ Book 2016; 35: 92-102. https://doi.org/10.14694/edbk_15922110.1200/ edbk_159221

[5] WU S, WU F, JIANG Z. Identification of hub genes, key miRNAs and potential molecular mechanisms of colorectal cancer. Oncol Rep 2017; 38: 2043-2050. https://doi.org/10.3892/ or.2017.5930

[6] WENG W, WEI Q, TODEN S, YOSHIDA K, NAGASAKA T et al. Circular RNA ciRS-7-A Promising Prognostic Biomarker and a Potential Therapeutic Target in Colorectal Cancer. Clin Cancer Res 2017; 23: 3918-3928. https://doi. org/10.1158/1078-0432.ccr-16-2541

[7] DU WW, ZHANG C, YANG W, YONG T, AWAN FM et al. Identifying and Characterizing circRNA-Protein Interaction. Theranostics 2017; 7: 4183-4191. https://doi.org/10.7150/ thno.21299

[8] LASDA E, PARKER R. Circular RNAs: diversity of form and function. RNA 2014; 20: 1829-1842. https://doi.org/10.1261/ rna.047126.114

[9] HANSEN TB, KJEMS J, DAMGAARD CK. Circular RNA and miR-7 in cancer. Cancer Res 2013; 73: 5609-5612. https://doi.org/10.1158/0008-5472.can-13-1568

[10] HSIAO KY, LIN YC, GUPTA SK, CHANG N, YEN L et al. Noncoding Effects of Circular RNA CCDC66 Promote Colon Cancer Growth and Metastasis. Cancer Res 2017; 77: 2339-2350. https://doi.org/10.1158/0008-5472.can-161883

[11] ZHANG X, WANG S, WANG H, CAO J, HUANG X et al. Circular RNA circNRIP1 acts as a microRNA-149-5p sponge to promote gastric cancer progression via the AKT1/mTOR pathway. Mol Cancer 2019; 18: 20. https://doi.org/10.1186/ s12943-018-0935-5

[12] CHEN Y, YE X, XIA X, LIN X. Circular RNA ABCB10 correlates with advanced clinicopathological features and unfavorable survival, and promotes cell proliferation while reduces cell apoptosis in epithelial ovarian cancer. Cancer Biomark 2019; 26: 151-161. https://doi.org/10.3233/CBM190064
[13] HUANG Y, ZHANG Y, JIA L, LIU C, XU F. Circular RNA ABCB10 promotes tumor progression and correlates with pejorative prognosis in clear cell renal cell carcinoma. Int J Biol Markers 2019; 34: 176-183. https://doi. org/10.1177/1724600819842279

[14] LIANG HF, ZHANG XZ, LIU BG, JIA GT, LI WL. Circular RNA circ-ABCB10 promotes breast cancer proliferation and progression through sponging miR-1271. Am J Cancer Res 2017; 7: 1566-1576.

[15] HE L, HANNON GJ. MicroRNAs: small RNAs with a big role in gene regulation. Nat Rev Genet 2004; 5: 522-531. https://doi.org/10.1038/nrg1379

[16] MEMCZAK S, JENS M, ELEFSINIOTI A, TORTI F, KRUEGER J et al. Circular RNAs are a large class of animal RNAs with regulatory potency. Nature 2013; 495: 333-338. https://doi.org/10.1038/nature11928

[17] XIE F, LI Y, WANG M, HUANG C, TAO D et al. Circular RNA BCRC-3 suppresses bladder cancer proliferation through miR-182-5p/p27 axis. Mol Cancer 2018; 17: 144. https://doi.org/10.1186/s12943-018-0892-Z

[18] LIU Z, ZHOU Y, LIANG G, LING Y, TAN W et al. Circular RNA hsa_circ_001783 regulates breast cancer progression via sponging miR-200c-3p. Cell Death Dis 2019; 10: 55. https://doi.org/10.1038/s41419-018-1287-1

[19] WU L, HUI H, WANG LJ, WANG H, LIU QF et al. MicroRNA-326 functions as a tumor suppressor in colorectal cancer by targeting the nin one binding protein. Oncol Rep 2015; 33: 2309-2318. https://doi.org/10.3892/or.2015.3840

[20] SORIA G, BEN-BARUCH A. The inflammatory chemokines CCL2 and CCL5 in breast cancer. Cancer Lett 2008; 267: 271-285. https://doi.org/10.1016/j.canlet.2008.03.018

[21] HOMEY B, MULLER A, ZLOTNIK A. Chemokines: agents for the immunotherapy of cancer Nat Rev Immunol 2002; 2: 175-184. https://doi.org/10.1038/nri748

[22] ALDINUCCI D, COLOMBATTI A. The inflammatory chemokine CCL5 and cancer progression. Mediators Inflamm 2014; 2014: 292376. https://doi.org/10.1155/2014/292376

[23] CAMBIEN B, RICHARD-FIARDO P, KARIMDJEE BF, MARTINI V, FERRUA B et al. CCL5 neutralization restricts cancer growth and potentiates the targeting of PDGFRbeta in colorectal carcinoma. PLoS One 2011; 6: e28842. https:// doi.org/10.1371/journal.pone.0028842

[24] GALLUZZI L, VITALE I, AARONSON SA, ABRAMS JM, ADAM D et al. Molecular mechanisms of cell death: recommendations of the Nomenclature Committee on Cell Death 2018. Cell Death Differ 2018; 25: 486-541. https://doi. org/10.1038/s41418-017-0012-4

[25] THOMPSON CB. Apoptosis in the pathogenesis and treatment of disease. Science 1995; 267: 1456-1462. https://doi. org/10.1126/science.7878464

[26] MOU Y, WANG J, WU J, HE D, ZHANG C et al. Ferroptosis, a new form of cell death: opportunities and challenges in cancer. J Hematol Oncol 2019; 12: 34. https://doi. org/10.1186/s13045-019-0720-y

[27] DIXON SJ, LEMBERG KM, LAMPRECHT MR, SKOUTA R, ZAITSEV EM et al. Ferroptosis: an iron-dependent form of nonapoptotic cell death. Cell 2012; 149: 1060-1072. https:// doi.org/10.1016/j.cell.2012.03.042 
[28] YIN Y, LONG J, HE Q, LI Y, LIAO Y et al. Emerging roles of circRNA in formation and progression of cancer. J Cancer 2019; 10: 5015-5021. https://doi.org/10.7150/jca.30828

[29] SHEN T, CHENG X, LIU X, XIA C, ZHANG H et al. Circ_0026344 restrains metastasis of human colorectal cancer cells via miR-183. Artif Cells Nanomed Biotechnol 2019; 47: 4038-4045. https://doi.org/10.1080/21691401.2019.1669 620

[30] PEI FL, CAO MZ, LI YF. Circ_0000218 plays a carcinogenic role in colorectal cancer progression by regulating miR-1393p/RAB1A axis. J Biochem 2020; 167: 55-65. https://doi. org/10.1093/jb/mvz078

[31] LU C, JIANG W, HUI B, RONG D, FU $\mathrm{K}$ et al. The circ_0021977/miR-10b-5p/P21 and P53 regulatory axis suppresses proliferation, migration, and invasion in colorectal cancer. J Cell Physiol 2020; 235: 2273-2285. https://doi. org/10.1002/jcp.29135

[32] GREEN DR, GALLUZZI L, KROEMER G. Cell biology. Metabolic control of cell death. Science 2014; 345: 1250256. https://doi.org/10.1126/science.1250256
[33] DAI X, KAUSHIK AC, ZHANG J. The Emerging Role of Major Regulatory RNAs in Cancer Control. Front Oncol 2019; 9: 920. https://doi.org/10.3389/fonc.2019.00920

[34] LV S, ZHANG J, HE Y, LIU Q, WANG Z et al. MicroRNA520e targets AEG-1 to suppress the proliferation and invasion of colorectal cancer cells through Wnt/GSK-3beta/beta-catenin signaling. Clin Exp Pharmacol Physiol 2020; 47: 158-167. https://doi.org/10.1111/1440-1681.13185

[35] ZHANG DL, YANG N. MiR-3174 functions as an oncogene in rectal cancer by targeting PCBD2. Eur Rev Med Pharmacol Sci 2019; 23: 2417-2426. https://doi.org/10.26355/eurrev_201903_17388

[36] TADA N, TSUNO NH, KAWAI K, MURONO K, NIREI T et al. Changes in the plasma levels of cytokines/chemokines for predicting the response to chemoradiation therapy in rectal cancer patients. Oncol Rep 2014; 31: 463-471. https://doi. org/10.3892/or.2013.2857 